\title{
Graph-Based Resource Allocation for D2D Communications Underlying Cellular Networks in Multiuser Scenario
}

\author{
Bin Guo, ${ }^{1}$ Shaohui Sun, ${ }^{1,2}$ and Qiubin Gao ${ }^{2}$ \\ ${ }^{1}$ School of Electrical and Information Engineering, Beihang University, Beijing, China \\ ${ }^{2}$ State Key Laboratory of Wireless Mobile Communications, China Academy of Telecommunications Technology (CATT), \\ Beijing, China
}

Correspondence should be addressed to Bin Guo; guobin.buaa@gmail.com

Received 12 May 2014; Revised 15 July 2014; Accepted 16 July 2014; Published 12 August 2014

Academic Editor: Lingyang Song

Copyright (C) 2014 Bin Guo et al. This is an open access article distributed under the Creative Commons Attribution License, which permits unrestricted use, distribution, and reproduction in any medium, provided the original work is properly cited.

With the characteristics of considerable user equipment, massive traffic and numerous local services, but limited frequency resources, the mobile communications in the future require efficient use of frequency resources. Device-to-device (D2D) communications underlying cellular networks have been considered as a promising solution to improve the resources utilization in local scenario. In this paper, we investigated the resources allocation problem in multiuser scenario and proposed a graph-based resources allocation scheme which can achieve suboptimal performance but with low computational complexity and less feedback information.

\section{Introduction}

The development of mobile communications is rapid. ITU forecasted that the amount of global mobile user equipment (UE) will catch up with the world's total population in 2014 [1]. Informa Telecoms \& Media Company estimated that the global mobile data traffic will reach 39.75 trillion MB in 2016, which is 10 times the traffic in 2011 [2]. Furthermore, according to a 3GPP's report [3], about 80 to $90 \%$ of the system throughput will occur in local scenarios in future, such as hotspot scenarios and indoor scenarios. These forecasts show that the future mobile communications are characterized by considerable UE, massive traffic, and numerous local services.

In addition, at the World Radio Communication Conference in 2007, the bandwidth allocated to mobile communications is less than $600 \mathrm{MHz}$ [4]. However, ITU predicted that the bandwidth requirement for mobile communications will reach $1280-1720 \mathrm{MHz}$ in 2020 [5]. On one hand there are increasing demands, and on the other hand there is a lack of frequency resources. This conflict puts forward the challenge for efficient use of frequency resources.

D2D communications as short-range communication technologies underlying cellular networks can not only improve the transmission rate and save propagation latency and transmission power, but also effectively improve the spectrum efficiency and the system performance [6, 7]. Therefore, they are considered as a promising solution to local services.

A key issue of D2D communications underlying cellular networks is the resource allocation and there have been a lot of researches about this issue [8-11]. In [8], the optimum resources allocation and power control are analyzed, and the results show that, by proper resources management, D2D communications can effectively improve the total throughput with limited interference between cellular links and D2D communications. In [9], an interference-aware resource allocation scheme is proposed to minimize the interference and obtain substantial gains in system performance. In [10], a novel resource allocation method by which D2D can reuse the resources of more than one cellular user is proposed and the selection of the optimal resource allocation method is discussed. The resource allocation problem was formulated as a noncooperative resource allocation game in [11], and an efficient auction algorithm was proposed to improve the performance of D2D communication. The simulation results showed that the proposed algorithm has close performance to 
the centralized scheme, and UE battery lifetime can be greatly extended.

However, these studies assumed that the number of D2D pairs is not greater than the number of channel resources, which ignores that the number of D2D pairs will exceed the number of available resources in future. In a multiuser scenario, in order to make users as many as possible to get reliable service, it is inevitable to share channel resources between different D2D pairs. Therefore, the interference situation in system will become more complicated. Except for the interferences between D2D communications and cellular networks, there are additional interferences between different D2D pairs. In addition, the channel state information (CSI) required for interference management at BS will increase, which will increase BS's burden.

In this paper, we propose a graph-based resource allocation scheme for D2D communications in multiuser scenario to solve the above problems. Graph theory is an effective mathematical tool, which is widely used to model and analyze the interaction and relationship of different types of networks. There have been some schemes using graph theory to allocate resources for D2D communications [12, 13]. In [12], an interference-aware graph-based resource sharing algorithm is proposed in downlink scenario of $\mathrm{D} 2 \mathrm{D}$ communications underlying cellular networks. The algorithm can effectively obtain the near optimal resource assignment solutions at the BS but with low computational complexity. However, the interference-awareness is defined as a condition that the BS can acquire local awareness on channel gains of each communication link and interference link. With the number of D2D pairs increasing, the graph will become complicated. In [13], a weighted bipartite graph-based scheme is proposed in uplink scenario. This scheme divides pieces of cellular UE and D2D pairs into two parties and converts the resources allocation problem into a matching problem. Matching is processed according to the weight which is defined as the difference between the channel capacity of the D2D pair and the cellular UE and the channel capacity of the cellular UE without D2D pair. The proposed channel sharing scheme increases the system capacity. However, the scheme assumes that the number of D2D pairs is not greater than the number of channel resources. For the opposite case, the paper proposes taking the cellular users as the matching object, which may affect the quality of cellular communications.

To our knowledge, there have been no researches for D2D communications based on graph theory in multiuser scenario. In this paper, the proposed scheme can reduce computational complexity and the amount of required feedback information on the premise of keeping guaranteed system performance.

The rest of this paper is organized as follows. In Section 2, the $\mathrm{D} 2 \mathrm{D}$ communications underlying cellular networks in multiuser scenario are described and the resource sharing problem is formulated. In Section 3, the graph-based resources allocation scheme is proposed. In Section 4, we show the simulation results and present analysis on the simulation results. In Section 5, the conclusions are drawn.

\section{System Model}

2.1. System Model. As illustrated in Figure 1, we consider an uplink transmission scenario in a cellular network, in which pieces of cellular UE transmit signals to BS. It is assumed that there are $M$ traditional pieces of cellular UE (indicated by $\left.U_{m}\right)$ and $N(N \geq M)$ D2D pairs (indicated by $D_{n}$ ) within the coverage of BS. Pieces of cellular UE and $\mathrm{D} 2 \mathrm{D}$ pairs are uniformly distributed in the area. D2D transmitters (indicated by $\mathrm{DT}_{n}$ ) transmit signals to $\mathrm{D} 2 \mathrm{D}$ receiver (indicated by $\mathrm{DR}_{n}$ ) and the maximum distance between them is $D$.

BS allocates channel resources to cellular communications and D2D communications. It is assumed that channel resources are orthogonal, and the interference only exists in intrachannel when different links share the same channel resource. Given that the number of channel resources equals the number of pieces of cellular UE, that is, being equal to $M$, one D2D pair is allowed to share one channel resource with other $\mathrm{D} 2 \mathrm{D}$ pairs. Therefore, there are two kinds of interferences in this scenario due to channel resources sharing. One is the interference between cellular links and D2D communications. Another is the interference between different D2D pairs.

In order to reduce interference and save energy, BS controls the transmit power of pieces of cellular UE and D2D transmitters determine transmit power themselves by setting the maximum transmit power $\mathrm{PT}_{\max }$ and the maximum received power $\mathrm{PR}_{\max }$. In general, transmitters will transmit signals in $\mathrm{PT}_{\max }$. However, when a received power exceeds the $\mathrm{PR}_{\max }$, the corresponding transmitter should reduce the transmit power.

In this paper, the channel model is considered as Rayleigh fading channel, and the channel response follows the independent complex Gaussian distribution. Besides, the path loss model is considered as distance-dependent path loss. Therefore, the channel gains contain the normalized smallscale fading and the path loss. We use $g_{\mathrm{U}_{m}, \mathrm{BS}}, g_{\mathrm{DT}_{n}, \mathrm{DR}_{n}}, g_{\mathrm{DT}_{n}, \mathrm{BS}}$, and $g_{U_{m}, \mathrm{DR}}$ to, respectively, represent the channel gains of the traditional cellular communication link from $U_{m}$ to BS, the $\mathrm{D} 2 \mathrm{D}$ communication link from $\mathrm{DT}_{n}$ to $\mathrm{DR}_{n}$, the interference link from $\mathrm{DT}_{n}$ to $\mathrm{BS}$, and the interference link from $U_{m}$ to $\mathrm{DR}_{n}$.

Consider the following:

$$
\begin{gathered}
g_{U_{m}, \mathrm{BS}}=\sqrt{P L_{U_{m}, \mathrm{BS}}} h_{U_{m}, \mathrm{BS}}, \\
g_{\mathrm{DT}_{n}, \mathrm{DR}_{n}}=\sqrt{P L_{\mathrm{DT}_{n}, \mathrm{DR}_{n}}} h_{\mathrm{DT}_{n}, \mathrm{DR}}, \\
g_{\mathrm{DT}_{n}, \mathrm{BS}}=\sqrt{P L_{\mathrm{DT}_{n}, \mathrm{BS}}} h_{\mathrm{DT}_{n}, \mathrm{BS}}, \\
g_{U_{m}, \mathrm{DR}}=\sqrt{P L_{U_{m}, \mathrm{DR}}} h_{U_{m}, \mathrm{DR}},
\end{gathered}
$$

where $P L_{U_{m}, \mathrm{BS}}, P L_{\mathrm{DT}_{n}, \mathrm{DR}_{n}}, P L_{\mathrm{DT}_{n}, \mathrm{BS}}$, and $P L_{U_{m}, \mathrm{DR}_{n}}$ are the

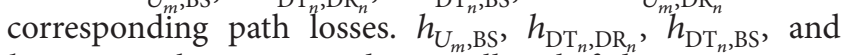
$h_{U_{m}, \mathrm{DR}_{n}}$ are the corresponding small-scale fading.

2.2. Problem Formulation. We consider that cellular communications have higher priority and BS must guarantee 


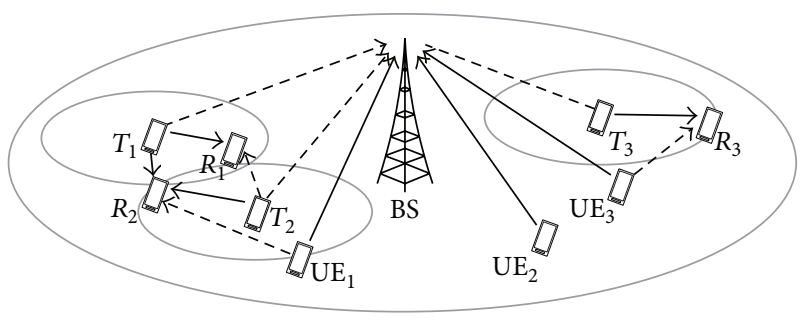

$\longrightarrow$ Signal

$-\rightarrow$ Interference

Figure 1: System model for D2D communications underlying cellular network when sharing uplink resource.

the performance of cellular links first. Therefore, resources allocation for D2D communications is considered under the assumption that all pieces of cellular UE have already equally obtained channel resources.

In multiuser scenario, in order to make $\mathrm{D} 2 \mathrm{D}$ pairs as many as possible obtaining effective resources, it is inevitable to allocate one channel resource to multiple D2D pairs. On one hand, that leads to a cumulative effect of the interference in one channel resource. On the other hand, that introduces additional interference between these D2D pairs. Therefore, in order to reduce the total interference level in the system as much as possible, it is required to conduct reasonable resources allocation. In this paper, we take the minimum interference level of the system as objective.

The resources sharing situation between pieces of cellular UE and D2D pairs are denoted as matrix $A_{M \times N}=\left[a_{m, n}\right]$, where $a_{m, n}=1$ and $a_{m, n}=0$ are, respectively, used to imply whether cellular UE $U_{m}$ and D2D pair $D_{n}$ share the same resources or not.

Thus, the objective can be expressed as

$$
\begin{array}{ll}
\min & \sum_{m=1}^{M} I_{m} \\
\text { s.t. } & \sum_{m=1}^{M} \alpha_{m, n}=1, \quad n=1, \ldots, N .
\end{array}
$$

The constraint guarantees that each D2D pair is allowed to obtain one channel resource.

$I_{m}$ is the interference power in channel resource $m$ :

$$
I_{m}=\sum_{m=1}^{M} \sum_{n=1}^{N}\left(I_{m, n}^{c}+I_{m, n}^{d}\right)
$$

where $I_{m, n}^{c}$ and $I_{m, n}^{d}$, respectively, represent the received interference at BS in channel $m$ from D2D pair $D_{n}$ and the received interference at $\mathrm{D} 2 \mathrm{D}$ pair $D_{n}$ in channel $m$ from cellular UE $U_{m}$ :

$$
\begin{gathered}
I_{m, n}^{c}=\alpha_{m, n} P_{\mathrm{DT}_{n}} g_{\mathrm{DT}_{n}, \mathrm{BS}}, \\
I_{m, n}^{d}=\alpha_{m, n} P_{U_{m}} g_{U_{m}, \mathrm{DR}_{n}}+\sum_{\substack{i=1 \\
i \neq n}}^{N} \alpha_{m, i} P_{\mathrm{DT}_{i}} g_{\mathrm{DT}_{i}, \mathrm{DR}_{n}},
\end{gathered}
$$

where $P_{\mathrm{DT}_{n}}$ is the transmit power of $\mathrm{DT}_{n}$.
Thus, the optimization objective can be rewritten as

$$
\begin{aligned}
& \min \sum_{m=1}^{M} I_{m} \\
&=\min \sum_{m=1}^{M} \sum_{n=1}^{N}\left(\begin{array}{l}
\alpha_{m, n}\left(P_{\mathrm{DT}_{n}} g_{\mathrm{DT}_{n}, \mathrm{BS}}+P_{U_{m}} g_{U_{m}, \mathrm{DR}_{n}}\right) \\
\left.+\sum_{\substack{i=1 \\
i \neq n}}^{N} \alpha_{m, i} P_{\mathrm{DT}_{i}} g_{\mathrm{DT}_{i}, \mathrm{DR}_{n}}\right)
\end{array}\right.
\end{aligned}
$$

$$
\text { s.t. } \quad \sum_{m=1}^{M} \alpha_{m, n}=1, \quad n=1, \ldots, N \text {. }
$$

Moreover, in ideal conditions, BS should know CSIs of all communication links and all interference links to allocate resources. The information is reported by users. In multiuser scenario, BS not only considers the resources sharing between pieces of cellular UE and D2D pairs, but also considers the resources sharing between different D2D pairs. Therefore, a D2D pair needs to report CSIs of the links from all pieces of cellular UE and all the other D2D pairs. With the number of D2D pairs increasing, the amount of feedback information will be considerable. Hence, it is necessary to design an effective method to reduce the amount of feedback information.

\section{Graph-Based Resource Allocation Scheme}

In this section, the proposed graph-based resource allocation scheme is introduced in detail. In order to decrease the interference level of the system, we propose a resources allocation scheme giving priority to the resources with the minimum interference. In order to avoid the severe interference between D2D pairs and reduce the amount of feedback information, we set up interference matrixes based on a new feedback model. In addition, in order to ensure the performance of cellular links, we design a resource selection scheme to avoid too many D2D pairs reusing one cellular resource.

3.1. Feedback Model. Before allocating resources, BS collects CSIs of related channels. Hence, a D2D pair is required to have the ability of measuring channel state including the channels from pieces of cellular UE to the D2D receiver and the channels from all the other D2D transmitters to the D2D receiver and the ability of reporting the information to BS.

The channel state from pieces of cellular UE to D2D receivers can be measured and reported by $\mathrm{D} 2 \mathrm{D}$ receivers which monitor all communication channels, while, to measure the channel state of interference links between different D2D pairs, we define an exclusive channel for D2D communications, denoted by $\mathrm{D} 2 \mathrm{DCH}$, which consisted of $N$ orthogonal subchannels. The pattern of the subchannels can be multiple OFDM subcarriers, orthogonal spread spectrum codes, or independent time slots. Each subchannel matches a unique D2D pair. Each D2D transmitter launches its ID 


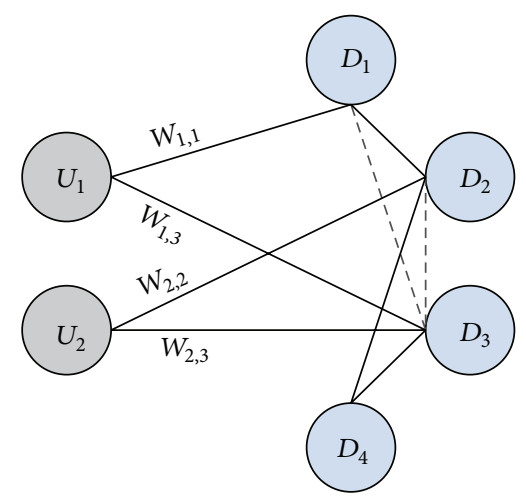

FIgURE 2: An illustrative example of the graph.

signal in corresponding subchannel and each $\mathrm{D} 2 \mathrm{D}$ receiver monitors the $\mathrm{D} 2 \mathrm{DCH}$ and reports the results to $\mathrm{BS}$.

It is seen that the required feedback information of one D2D pair includes CSIs of channels between this D2D pair and all pieces of cellular UE and CSIs of channels between this D2D pair and all the other D2D pairs. With the number of D2D pairs increasing, this feedback model will cause considerable overheads. Therefore, we design a new feedback model to reduce the overheads.

First of all, a threshold of interference power $\eta$ is set for $\mathrm{D} 2 \mathrm{D}$ communications. Then, D2D receivers monitor all cellular resources and report feedback information of those resources in which the received interference power is lower than $\eta$. After receiving the feedback information, BS establishes a matrix denoted as $X 1_{M \times N}=\left[x 1_{m, n}\right]$, where $x 1_{m, n}=1$ is used to imply that the cellular UE $U_{m}$ does not cause severe interference to D2D pair $D_{n}$ and cellular resource $m$ is available and $x 1_{m, n}=0$ implies that cellular resource $m$ is not available.

At the same time, D2D receivers monitor the subchannels of $\mathrm{D} 2 \mathrm{DCH}$ and record those subchannels in which the received interference power is larger than $\eta$. D2D receivers establish matrix denoted as $X 2_{N \times N^{\prime}}=\left[x 2_{n, n^{\prime}}\right]$, where the $x 2_{n, n^{\prime}}=1$ and $x 2_{n, n^{\prime}}=0$ are, respectively, used to imply whether the D2D pair $D_{n^{\prime}}$ will cause severe interference or not to D2D pair $D_{n}$ when they share the same channel resource. Every D2D receiver just needs to report its corresponding row in $X 2_{N \times N^{\prime}}$ to BS.

By this feedback model, D2D pairs just report a part of CSIs and a list, instead of CSIs of all channels. Thus, the amount of feedback information can be reduced considerably. Notice that do not set $\eta$ too small, or there will be some D2D pairs that cannot acquire resources. The way to set up a reasonable $\eta$ needs further study. Furthermore, with the matrix $X 2_{N \times N^{\prime}}$, BS can avoid allocating the same resource to D2D pairs which may cause severe interference.

3.2. Graph Construction. The first step of the graph-based resource allocation scheme is the graph construction. Here, we consider that a weighted bipartite graph which contains two parties of vertices, respectively, represents the pieces of cellular UE and D2D pairs, and some weighted edges represent the relationships between vertices. According to the scenario in Figure 1, the constructed graph is shown in Figure 2.

There are two pieces of cellular UE and four D2D pairs are in the graph. $U_{1}$ and $U_{2}$, which present the pieces of cellular UE, compose the left part, and $D_{1}, D_{2}, D_{3}$, and $D_{4}$, which present $\mathrm{D} 2 \mathrm{D}$ pairs, compose the right part. The graph is denoted by $G=\left(V^{c}, V^{d}, E\right)$, where $V^{c}$ is the vertices set of pieces of cellular UE, $V^{d}$ is the vertices set of D2D pairs, and $E$ is the edges set. Each vertex $v_{m}^{c} \in V^{c}$ represents a cellular UE and each vertex $v_{n}^{d} \in V^{d}$ represents a D2D pair. The edge $e_{m, n} \in E$ implies that the D2D pair $v_{n}^{d}$ shares the channel resource with the cellular UE $v_{m}^{c}$. Moreover, the weights set is denoted by $W_{M \times N}$, which is $M$-by- $N$ matrix. The element $w_{m, n} \in W_{M \times N}$, representing the weight of the $e_{m, n}$, equals the interference power $I_{m, n}^{c}$. In addition, the edge $e_{n, n^{\prime}} \in E$ connects $v_{n}^{d} \in V^{d}$ and $v_{n^{\prime}}^{d} \in V^{d}$, which implies the interference level between D2D pair $v_{n}^{d}$ and D2D pair $v_{n^{\prime}}^{d}$. When they will cause strong interference, the edge is denoted by dotted lines. When the interference can be ignored, the edge is denoted by solid lines. The interference level can be obtained according to the matrix $X 2_{N \times N^{\prime}}$.

3.3. Graph-Based Resource Allocation Scheme. The algorithm of the proposed scheme is detailed in Algorithm 1. Firstly, the graph is established and the parameters of the graph are initialized. The matrix $X 1_{M \times N}$ and matrix $X 2_{M \times N}$ are established according to the feedback information of each D2D pair. The $W_{M \times N}$ is calculated according to $X 1_{M \times N}$ and the edge between different D2D pairs is determined by $X 2_{M \times N}$. A list $L A_{1 \times M}$ is established to accumulate interference from allocated $\mathrm{D} 2 \mathrm{D}$ pairs on each channel resource and its elements are initialized to 0 . A list $L R_{1 \times N}$ is established to record the allocated resource for $\mathrm{D} 2 \mathrm{D}$ pairs and its elements are initialized to 0 .

Resources allocation is achieved by an iteration algorithm which is controlled by $L R_{1 \times N}$. When $L R_{1 \times N}$ does not have 0 elements, the calculation will be terminated. In order to reduce the interference level of the system, the resources with the minimum weight are allocated preferentially. However, only considering the weight may lead to a condition that too many $\mathrm{D} 2 \mathrm{D}$ pairs reuse one channel resource, which will cause strong accumulative interference on this cellular link. Therefore, we take the sum of weight and accumulated interference as the basis for selecting reuse resource. In each iteration, the edge with the minimum sum value is selected. Then, the selected weight is accumulated to $L A_{1 \times M}$. Resource allocation for D2D pair $D_{n}$ may affect D2D pairs whose value in the $n$th column of $X 2_{N \times N^{\prime}}$ is 1 . The allocated resource will be forbidden to share with those D2D pairs, so their weights associated with the allocated resource will be set to infinite. At this point, the allocation process for one D2D pair is completed. Repeat the above steps until each element of $L R_{1 \times N}$ is not 0 . 


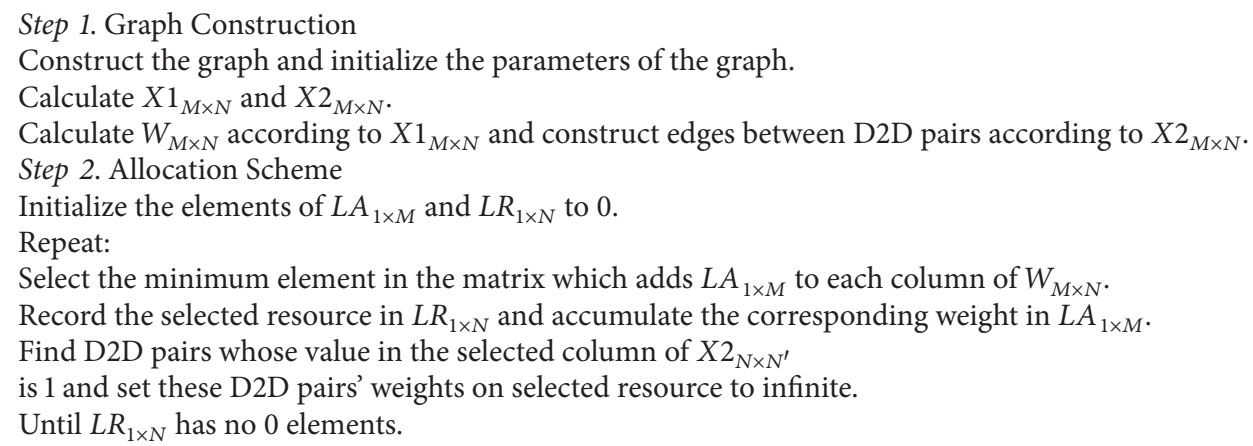

Algorithm 1: Graph-based resources allocation algorithm.

In addition, the transmit power of $\mathrm{D} 2 \mathrm{D}$ communications is determined by $\mathrm{D} 2 \mathrm{D}$ transmitters. The initial transmit power of a D2D pair is $\mathrm{PT}_{\max }$. According to the received power reported from D2D receiver, D2D transmitter computes the path loss. If the received power at receiver is lower than $\mathrm{PR}_{\max }$, the transmitter keeps transmit power. If the received power is larger than $P R_{\max }$, the transmitter computes a new transmit power according to the path loss. By power control, the interference can be further reduced.

3.4. Complexity Analysis. At last, we analyze the superiority of the proposed graph-based resource allocation scheme on computational complexity. According to the iteration algorithm in Algorithm 1, the computational complexity of the proposed scheme is relative to the initial state of the graph. In the worst case, the computational complexity is

$$
\chi_{\text {prop }}=O\left((M N)^{2}\right) \text {, }
$$

where the computational complexity of finding the minimum value from $M$-by- $N$ matrix is treated as $O(M N)$.

Meanwhile, the computational complexity of the enumeration scheme is calculated as

$$
\chi_{\text {enum }}=O\left(M^{N}\right) \text {. }
$$

It is obvious that as the number of D2D pairs increases, the computational complexity of the enumeration scheme will increase rapidly. However, the computational complexity of the proposed scheme is polynomial time. Therefore, the proposed scheme is more effective.

\section{Simulation Results and Analysis}

In this section, we give the simulation results of the proposed resources allocation scheme comparing with enumeration scheme, which achieves the optimal resources allocation through an exhaustive search, and random resources sharing scheme, which allocates the cellular resources to D2D pairs randomly. The parameters are shown in Table 1.

In terms of system interference, we compare three resource allocation schemes for the considered multiuser scenario of D2D communications underlying cellular network.
TABle 1: Parameters for simulation.

\begin{tabular}{lc}
\hline Parameter & Value \\
\hline Cell radius & $1000 \mathrm{~m}$ \\
The number of channel resources & 5 \\
Maximum D2D pair distance & $40 \mathrm{~m}$ \\
Maximum transmit power of pieces of cellular UE & $23 \mathrm{dBm}$ \\
Maximum transmit power of D2D transmitters & $23 \mathrm{dBm}$ \\
Maximum received power PR max $_{\text {Noise power }}$ & $-106 \mathrm{dBm}$ \\
SINR threshold of cellular links SINR & $-174 \mathrm{dBm}$ \\
Interference threshold of D2D communications $\eta$ & $6 \mathrm{~dB}$ \\
\hline
\end{tabular}

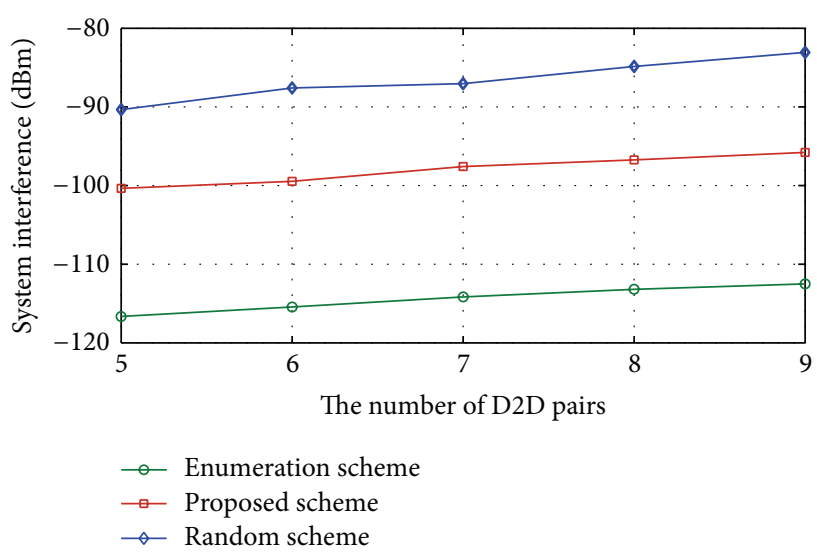

FIGURE 3: System interference with the number of D2D pairs.

Figure 3 shows that the system interference changed with the number of D2D pairs. It is shown that the interference level changed little with pair number. The enumeration scheme has the lowest interference level, the interference of the proposed scheme is bigger than the optimal scheme by $16 \mathrm{~dB}$, and the interference of the random scheme is the largest.

Moreover, we investigate the performance of system capacity. Figure 4 shows that, with different number of D2D pairs, the enumeration scheme achieves the optimal performance and the proposed scheme achieves suboptimal performance. However, the computational complexity of 


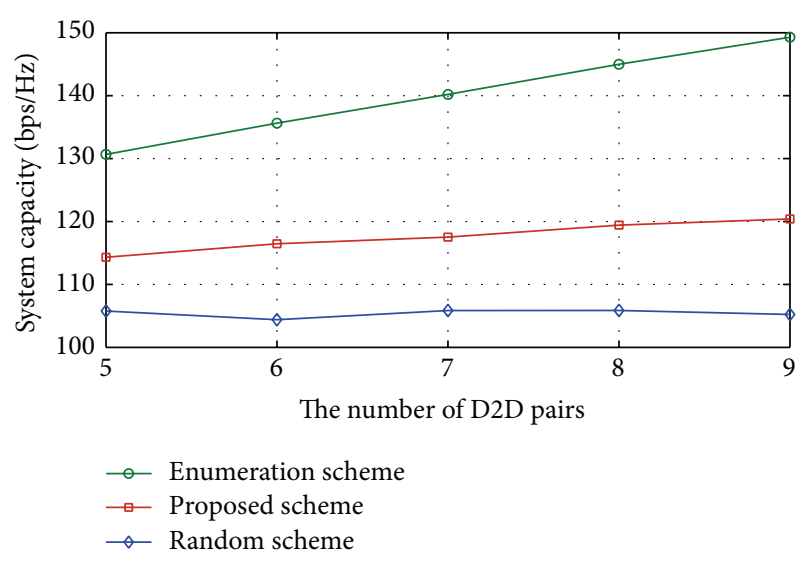

FIGURE 4: System capacity with the number of D2D pairs.

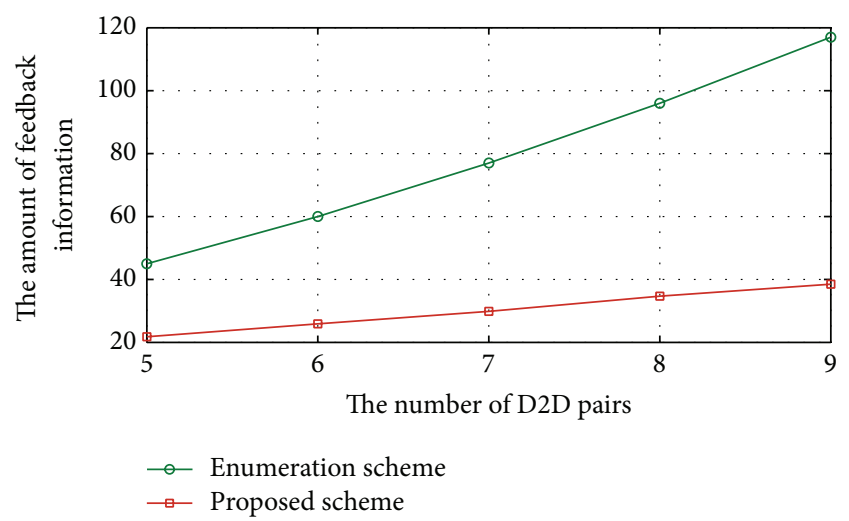

FIGURE 5: The average feedback amount with the number of D2D pairs.

the proposed scheme is much lower than the enumeration scheme. On the other hand, compared with the random scheme, the proposed scheme has a significant performance promotion in system capacity. In sum, we can conclude that the proposed scheme is an efficiency method for resources allocation.

Figure 5 shows that the average feedback information of all D2D pairs in system changed with the number of D2D pairs. As it is shown, the feedback amount of the enumeration scheme is much higher than the proposed scheme, and the difference between these two schemes is becoming larger with the number of D2D pairs increasing. It is indicated that the feedback model can not only help construct the edge between $\mathrm{D} 2 \mathrm{D}$ pairs, but also effectively reduce the amount of feedback information. Therefore, the proposed scheme will not increase overhead to the system.

\section{Conclusions}

In this paper, we proposed a resources allocation scheme based on graph theory for D2D communication underlying cellular networks in multiuser scenario. Simulation results show that the proposed resources allocation scheme achieves a lower interference level which is close to the optimal scheme but much lower than the random scheme. Furthermore, the proposed scheme can achieve suboptimal system capacity with much lower computational complexity than the enumeration scheme. At the same time, the proposed scheme sharply decreases the amount of feedback information.

\section{Conflict of Interests}

The authors declare that there is no conflict of interests regarding the publication of this paper.

\section{References}

[1] http://www.itu.int/en/Pages/default.aspx.

[2] Informa Telecoms \& Media, Mobile Content and Services Forecasts 2011-2016, 8th edition, 2012.

[3] 3GPP REV-080003, "Views for the LTE-Advanced requirements," Nokia, Nokia Siemens Networks.

[4] C. Yu, O. Tirkkonen, K. Doppler, and C. Ribeiro, "On the performance of device-to-device underlay communication with simple power control," in Proceedings of the IEEE Vehicular Technology Conference (VTC Spring), 5, p. 1, April 2009.

[5] "Estimated spectrum bandwidth requirements for the future development of IMT-2000 and IMT-Advanced," Report M.2078, ITU, 2000.

[6] D. Feng, L. Lu, Y.-Y. Wu, G. Y. Li, S. Li, and G. Feng, "Device-to-device communications in cellular networks," IEEE Communications Magazine, vol. 52, pp. 49-55, 2014.

[7] A. Asadi and V. Mancuso, "A Survey on Opportunistic Scheduling in Wireless Communications," IEEE Communications Surveys \& Tutorials, vol. 15, no. 4, pp. 1671-1688, 2013.

[8] C. Yu, K. Doppler, C. B. Ribeiro, and O. Tirkkonen, "Resource sharing optimization for device-to-device communication underlaying cellular networks," IEEE Transactions on Wireless Communications, vol. 10, no. 8, pp. 2752-2763, 2011.

[9] P. Jänis, V. Koivunen, Ć. Ribeiro, J. Korhonen, K. Doppler, and K. Hugl, "Interference-aware resource allocation for device-todevice radio underlaying cellular networks," in Proceedings of the IEEE 69th Vehicular Technology Conference (VTC '09), pp. 1-5, April 2009.

[10] B. Wang, L. Chen, X. Chen, X. Zhang, and D. Yang, "Resource allocation optimization for Device-to-Device communication underlaying cellular networks," in Proceeding of the 73rd IEEE Vehicular Technology Conference (VTC '11), pp. 1-6, Budapest, Hungary, May 2011.

[11] F. Wang, C. Xu, L. Song, Q. Zhao, X. Wang, and Z. Han, "Energy-aware resource allocation for device-to-device underlay communication," in Proceedings of the IEEE International Conference on Communications (ICC '13), pp. 6076-6080, 2013.

[12] R. Zhang, X. Cheng, L. Yang, and B. Jiao, "Interference-aware graph based resource sharing for device-to-device communications underlaying cellular networks," in Proceedings of the IEEE Wireless Communications and Networking Conference (WCNC '13), pp. 140-145, Shanghai, China, April 2013.

[13] H. Zhang, T. Wang, L. Song, and Z. Han, "Graph-based resource allocation for D2D communications underlaying cellular networks," in Proceedings of the IEEE/CIC International Conference on Communications in China-Workshops (CIC/ICCC '13), pp. 187-192, Xi'an, China, 2013. 

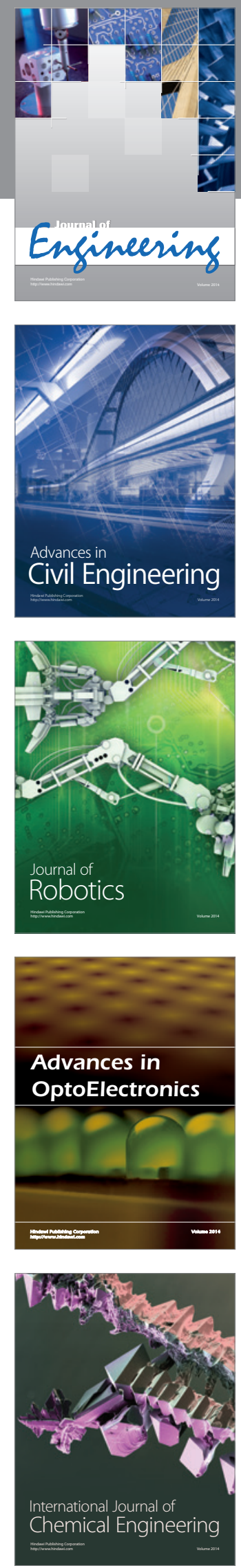

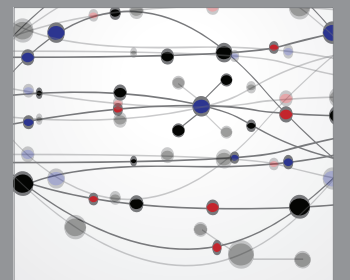

The Scientific World Journal
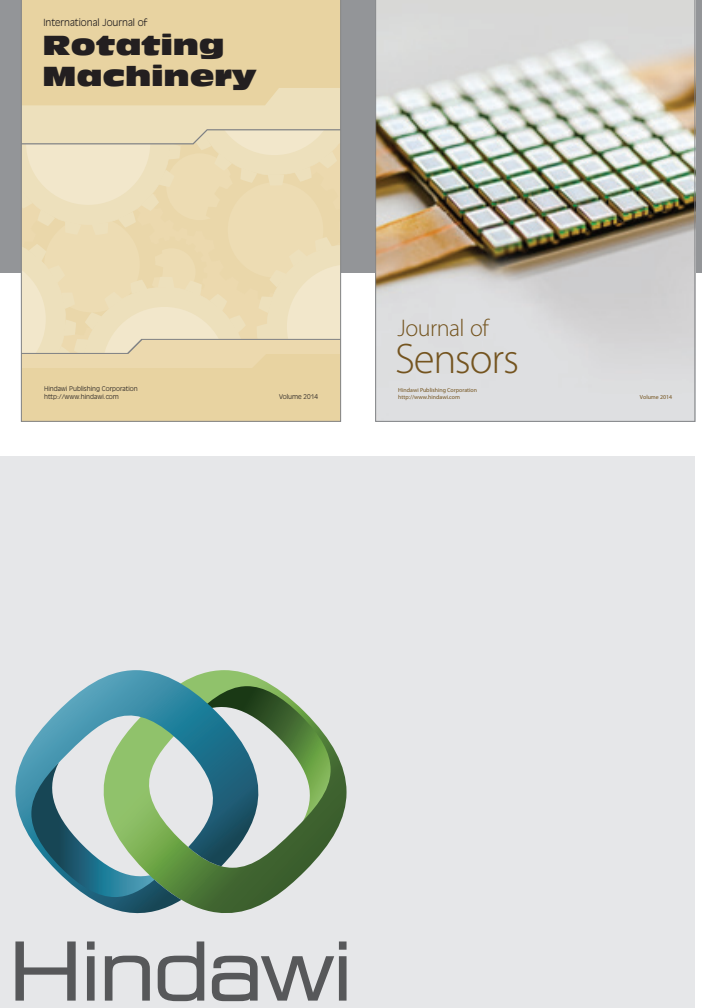

Submit your manuscripts at http://www.hindawi.com
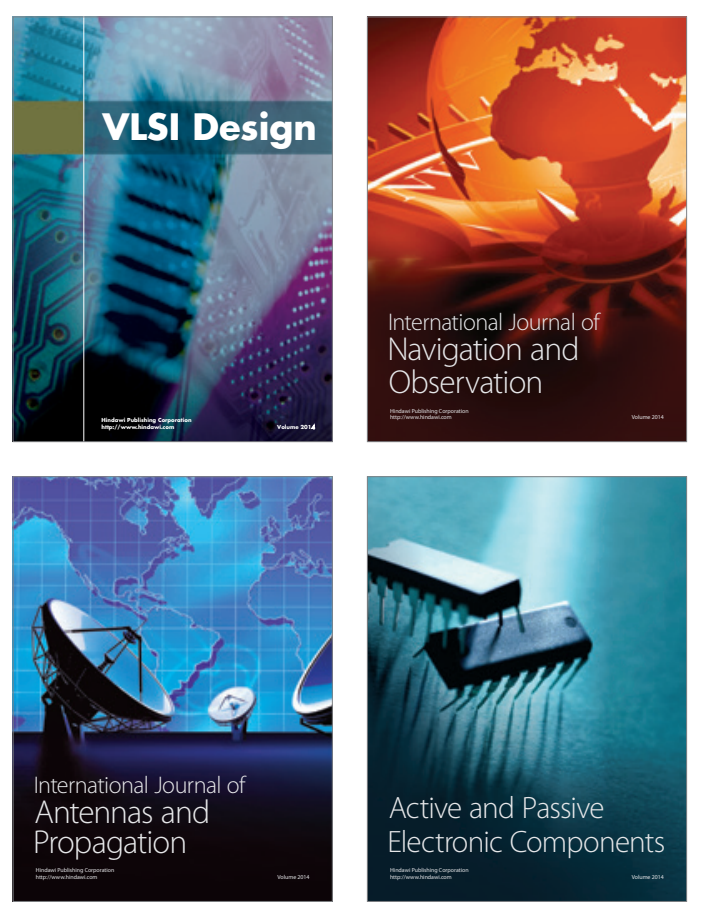
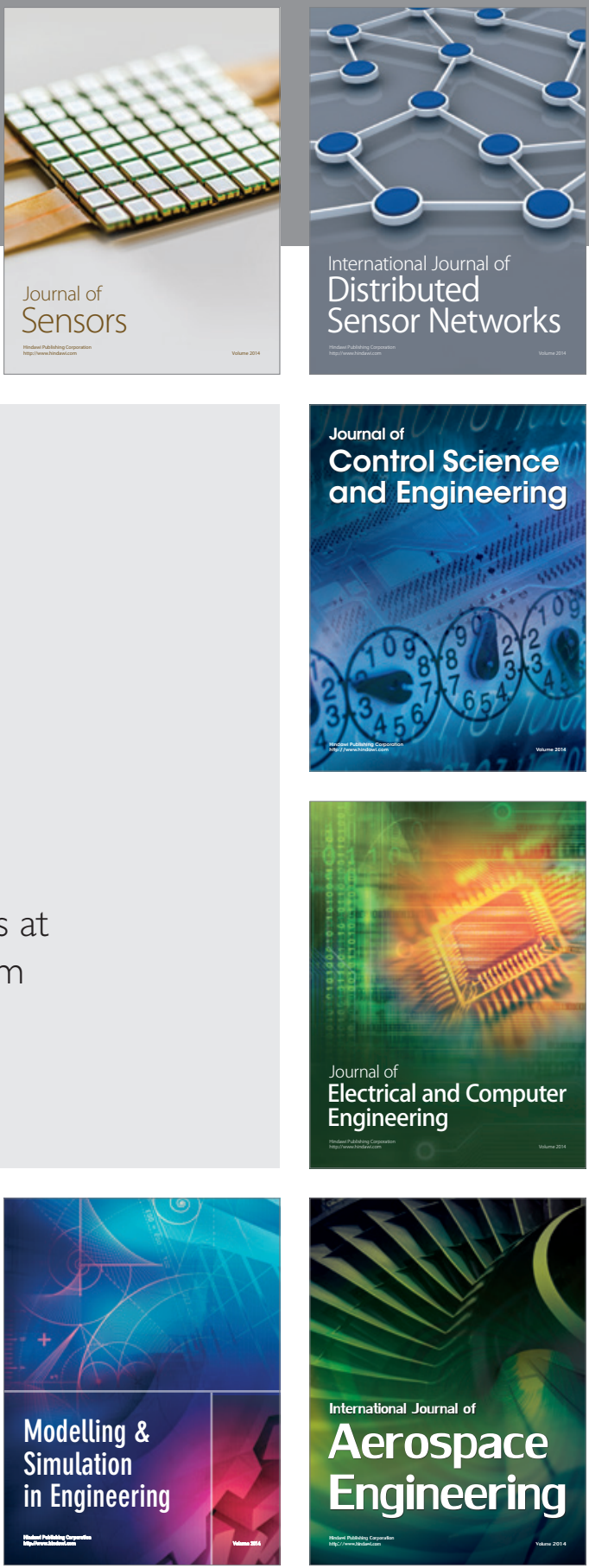

Journal of

Control Science

and Engineering
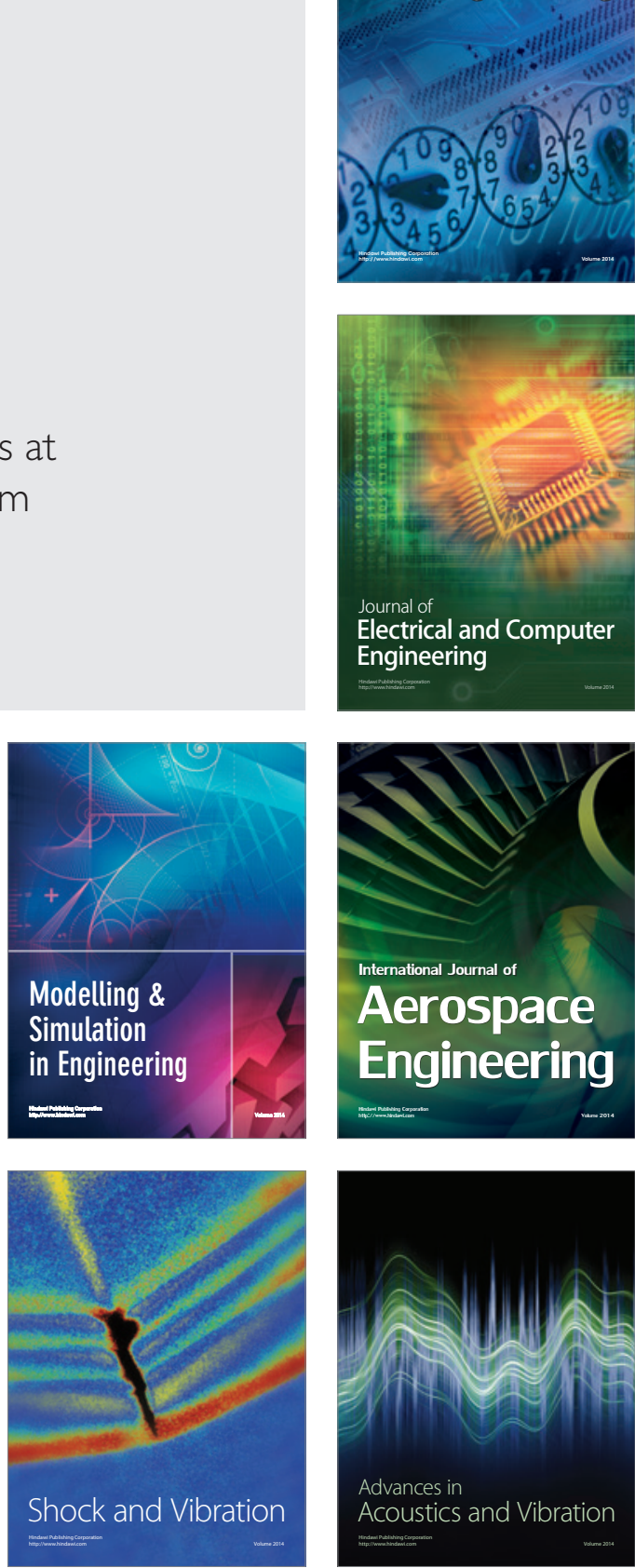